\title{
Fragment emission studies in low energy light heavy-ion reactions
}

\author{
T. K. Rana ${ }^{1, a}$, C. Bhattacharya ${ }^{1}$, S. Manna ${ }^{1}$, V. Srivastava ${ }^{1}$, K. Banerjee ${ }^{1}$, S. Kundu ${ }^{1}$, P. Roy ${ }^{1}$, R. Pandey ${ }^{1}$, A. \\ Chaudhuri $^{1}$, T. Roy ${ }^{1}$, T. K. Ghosh ${ }^{1}$, G. Mukherjee ${ }^{1}$, S. Bhattacharya ${ }^{1}$, J. K. Meena ${ }^{1}$, S. K. Pandit ${ }^{2}$, K. Mahata ${ }^{2}$, P. \\ Patale $^{2}$, A. Shrivastava ${ }^{2}$, and V. Nanal ${ }^{3}$ \\ ${ }^{1}$ Variable Energy Cyclotron Centre, Kolkata 700064, India \\ ${ }^{2}$ Nuclear Physics Division, Bhabha Atomic Research Centre, Mumbai - 400085, India \\ ${ }^{3}$ Tata Institute of Fundamental Research, Mumbai - 400005, India
}

\begin{abstract}
Fragment emission mechanisms have been studied in ${ }^{12} \mathrm{C}$ on ${ }^{12} \mathrm{C}$ and ${ }^{13} \mathrm{C}$ on ${ }^{12} \mathrm{C}$ reactions at same excitation energy. The inclusive energy distributions of the complex fragments $(3 \leq Z \leq 5)$ emitted from the composite system have been measured in the angular range $14^{0}$ to $36^{\circ}$. The present experiments have been performed with the motivation to study the isotopic dependence of fragment yields in these two reactions. From the preliminary analysis, it has been observed that fragments are emitted from a completely equilibrated and long lived composite system for both ${ }^{12} \mathrm{C}+{ }^{12} \mathrm{C}$ and ${ }^{13} \mathrm{C}+{ }^{12} \mathrm{C}$ reactions. It has also been observed that the emission of neutron-rich fragments are more in ${ }^{13} \mathrm{C}+{ }^{12} \mathrm{C}$ compared to ${ }^{12} \mathrm{C}+{ }^{12} \mathrm{C}$ reaction.
\end{abstract}

\section{Introduction}

Study of fragment emission mechanisms for light heavyions $\left(\mathrm{A}_{\text {proj. }}+\mathrm{A}_{\text {target }} \leq 60\right)$ collisions, at low energies $(\leq$ $10 \mathrm{MeV} / \mathrm{u}$ ), is a subject of great interest for many years [1]. The origin of these fragments extends from quasielastic, deep-inelastic transfer and orbiting to fusion - fission (FF) processes. The occurrence of FF and orbiting like processes have been observed experimentally in some light as well as medium heavy ion collision at beam energy well above the barrier [1-7]. The FF process appears to be less competitive in systems for which the number of open channels or available phase space in exit channel is less, however, a di-nuclear configuration survives for a longer period through an orbiting trajectory [2]. For reactions involving $\alpha$-clustered nuclei, e.g, ${ }^{20} \mathrm{Ne}+{ }^{12} \mathrm{C}$, ${ }^{24} \mathrm{Mg}+{ }^{12} \mathrm{C},{ }^{28} \mathrm{Si}+{ }^{12} \mathrm{C}$, etc., enhancement in the yield and/or resonance-like excitation function in a few outgoing channels (around the entrance channel) have indicated the role played by deep inelastic orbiting process in fragments emission [3-6]. In the FF mechanism, a completely equilibrated compound nucleus $(\mathrm{CN})$ is formed, which decays into various exit channels. The decay probability is governed by the available phase space and barrier penetration probabilities for the respective decay channels. However, deep inelastic orbiting may be described in terms of the formation of a long-lived, di-nuclear molecular complex [7], which acts as a "door way to fusion" with a strong memory of the entrance channel. In this picture, the interacting ions are trapped in a more deformed configuration than that of the compound nucleus (trapped in the pocket of the ion-ion interaction potential due to com-

\footnotetext{
ae-mail: tapan@vecc.gov.in
}

bined effects of Coulomb and centrifugal barriers). Both orbiting and fusion-fission processes occur on similar time scales. In addition to that, for the light heavy-ion systems, the shapes of the orbiting di-nuclear complexes are quite similar to the saddle and scission shapes obtained in course of evolution of the FF process. Therefore, it is difficult to differentiate the signatures of the two processes. Study of fragments emission mechanism is considered to be an important tool to study such entrance channel effects; however, such data are scarce at low energies.

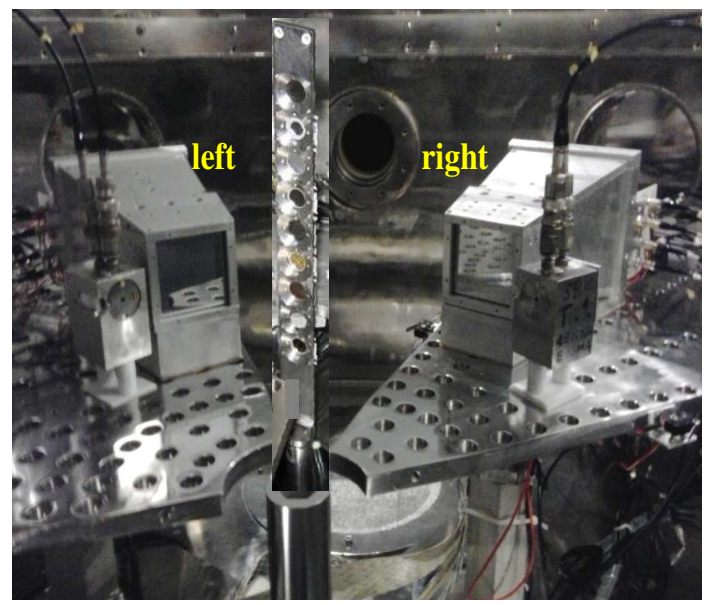

Figure 1. Typical experimental setup inside scattering chamber.

Here we report our measurements of fragments emission in ${ }^{12} \mathrm{C}(80 \mathrm{MeV})$ on ${ }^{12} \mathrm{C}$ and ${ }^{13} \mathrm{C}(78.5 \mathrm{MeV})$ on ${ }^{12} \mathrm{C}$ reactions. The energy of the beams $\left[{ }^{12} \mathrm{C}(80 \mathrm{MeV})\right.$ and 
$\left.{ }^{13} \mathrm{C}(78.5 \mathrm{MeV})\right]$ have been chosen to form the composite systems at same excitation energy. The ${ }^{12} \mathrm{C}+{ }^{12} \mathrm{C}$ reaction has been chosen as the number of open channels for this reaction is very small [2] and most likely to form long lived di-nuclear type composite system. In a recent study by Morelli et al. [8], residual deviations from the statistical behaviour have been seen in two specific exit channels (carbon with $3 \alpha$ 's and oxygen with $2 \alpha$ 's channels) for the reaction ${ }^{12} \mathrm{C}+{ }^{12} \mathrm{C}$, which have tentatively been assigned due to the presence of direct reactions and/or $\alpha$-clustering effects. Anomalously high branching ratios with respect to the statistical model calculation for these channels also have been observed [9]. The present experiments have been performed with the motivation to see the isotopic dependence of fragments yield in these two reactions.

\section{Experimental details}

The experiments have been performed at Pelletron-Linac facility, Mumbai, using $80 \mathrm{MeV}{ }^{12} \mathrm{C}$ and $78.5{ }^{13} \mathrm{C}$ ion beams on ${ }^{12} \mathrm{C}$ target (thickness $\sim 70 \mu \mathrm{g} / \mathrm{cm}^{2}$ ). The emitted fragments have been identified using two telescopes, each consisting of $\sim 50 \mu \mathrm{m} \Delta \mathrm{E}$ single-sided silicon strip detector (SSSD), $\sim 1000 \mu \mathrm{m}$ E double-sided silicon strip detector (DSSD) and backed by four CSI(Tl) detectors, each of thickness $6 \mathrm{~cm}$. Typical experimental setup has been shown in figure 1 . The angular resolutions were $0.8^{0}$ and $1^{0}$ for the right and left telescope in figure 1 , respectively. Typical two dimensional plots of $\Delta \mathrm{E}$ versus $\mathrm{E}$ particle identification spectra for strip detectors are shown in figure 2 and in figure 3 for ${ }^{12} \mathrm{C}+{ }^{12} \mathrm{C}$ and for ${ }^{13} \mathrm{C}+{ }^{12} \mathrm{C}$ reactions, respectively. Very good isotopic separation for different fragments have been observed. Inclusive energy distributions for various fragments $(3 \leq \mathrm{Z} \leq 5)$ have been measured in the angular range of $14^{0}$ to $36^{0}$. Energy calibration of the telescopes have been performed using elastically scattered ${ }^{12,13} \mathrm{C}$ ions from ${ }^{209} \mathrm{Bi}$ target at different energies $\left({ }^{12} \mathrm{C}\right.$ beam of 70 and $80 \mathrm{MeV},{ }^{13} \mathrm{C}$ beam of 78.5 and $82 \mathrm{MeV}$ ) and $\alpha$-particles from the ${ }^{229} \mathrm{Th} \alpha$-source.

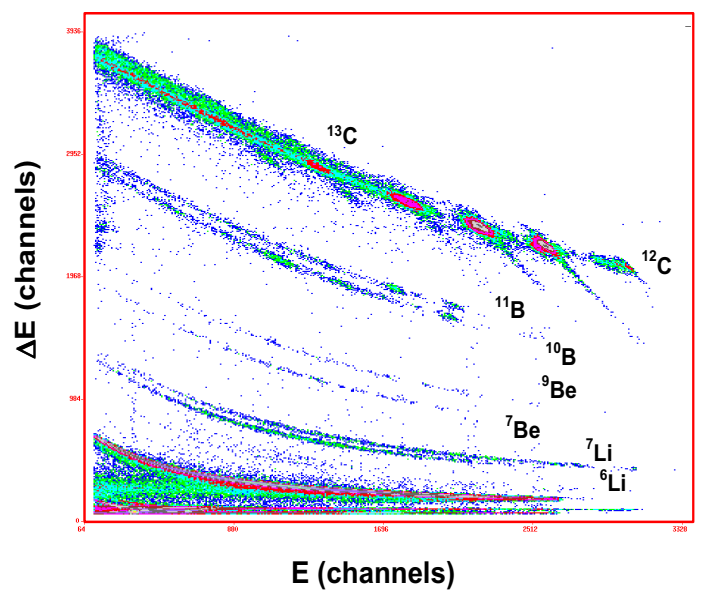

Figure 2. Typical two dimensional plot between thin Si-strip $(\Delta \mathrm{E}$ , $50 \mu \mathrm{m})$ and thick Si-strip (E, $1000 \mu \mathrm{m})$ detector for the ${ }^{12} \mathrm{C}+$ ${ }^{12} \mathrm{C}$ reaction.

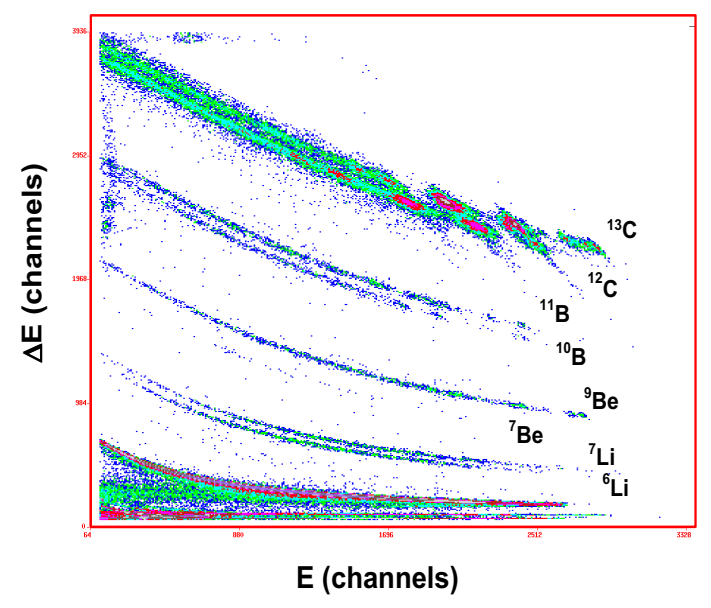

Figure 3. Same as figure 2 for the reaction ${ }^{13} \mathrm{C}+{ }^{12} \mathrm{C}$.

\section{Results and discussions}

Typical inclusive energy spectra $\left(\theta=14^{0}\right)$ of different isotopes of the fragments $\mathrm{Li}$, and $\mathrm{Be}$ obtained in the reactions ${ }^{12} \mathrm{C}+{ }^{12} \mathrm{C}$ and ${ }^{13} \mathrm{C}+{ }^{12} \mathrm{C}$ have been shown in figure 4 and figure 5 by solid and dotted lines respectively. The energy distributions are nearly Gaussian in shape (excluding the transfer channel), having their centroid at the expected kinetic energies for the fission fragments obtained from the Viola systematics corrected by the corresponding asymmetry factors [10] (shown by arrows in figure 4 and figure 5). This suggests that, in all cases, the fragments are emitted from a fully energy relaxed composite as expected for both FF and orbiting processes.

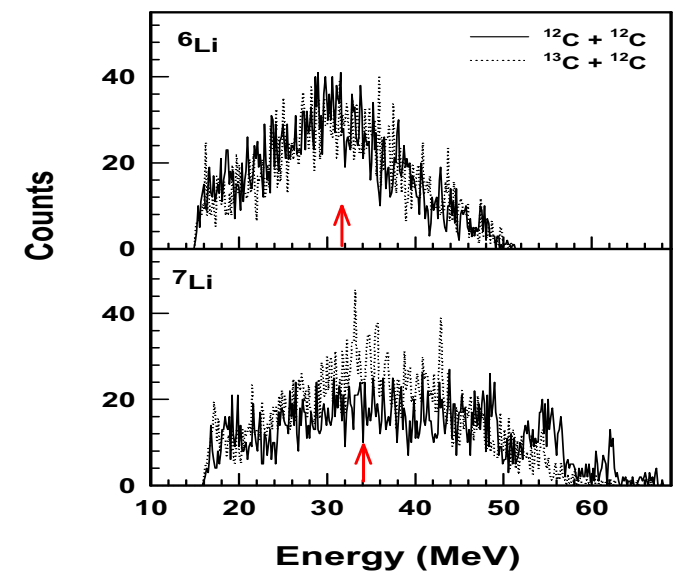

Figure 4. Typical energy spectra of isotopes of $\mathrm{Li}$ in the reactions ${ }^{12} \mathrm{C}+{ }^{12} \mathrm{C}$ (solid-line) and ${ }^{13} \mathrm{C}+{ }^{12} \mathrm{C}$ (dotted-line). Arrows indicate the energy of the fragments as obtained from Viola systematics corrected by asymmetric factor.

Angular distributions of different isotopes of $\mathrm{Li}$ and Be have been obtained by integrating the respective normalized energy distributions under a fitted Gaussian with a mean value obtained from Viola systematics corrected by asymmetric factor. The transformations from the laboratory to center-of-mass $(\mathrm{cm})$ system have been done with 


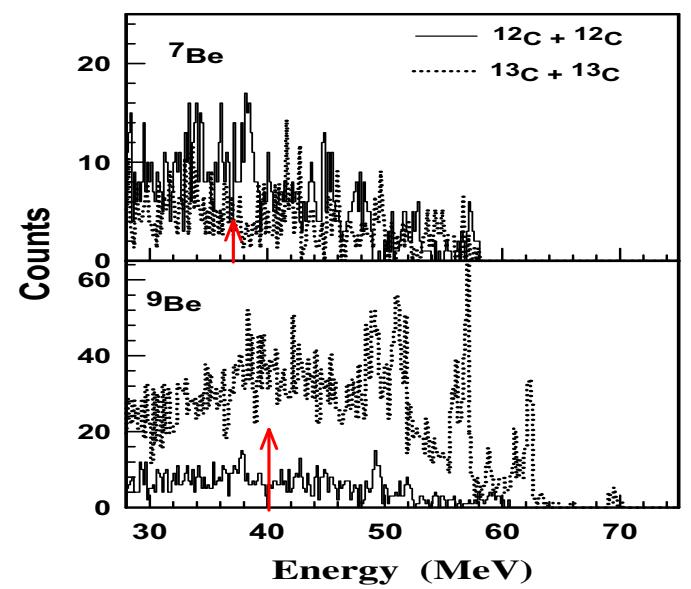

Figure 5. Typical energy spectra of emitted isotopes of $\mathrm{Be}$ in the reactions ${ }^{12} \mathrm{C}+{ }^{12} \mathrm{C}$ (solid-line) and ${ }^{13} \mathrm{C}+{ }^{12} \mathrm{C}$ (dotted-line). Arrows indicate the energy of the fragments as obtained from Viola systematics corrected by asymmetric factor.

the assumption of a two body kinematics averaged over total kinetic energy distributions. The $\mathrm{cm}$ angular distributions $(\mathrm{d} \sigma / \mathrm{d} \Omega)$, of different isotopes of $\mathrm{Li}$ and Be obtained in the above two reactions have been shown in figure 6 and figure 7, respectively. The angular distributions of all the fragments emitted in both the reactions are found to follow $\sim 1 / \sin \theta_{c m}$ dependence in $\mathrm{cm}$ (shown by solid lines (red) in figure 6 and figure 7), which further conjectured the emission of fragments are from a completely equilibrated, long-lived, composite system. It is seen from figure 6 and figure 7 that, at each angle, the differential cross-sections of neutron-rich fragments, viz. ${ }^{7} \mathrm{Li}$ and ${ }^{9} \mathrm{Be}$, obtained in ${ }^{13} \mathrm{C}+{ }^{12} \mathrm{C}$ reaction are more than those obtained in ${ }^{12} \mathrm{C}+$ ${ }^{12} \mathrm{C}$ reaction. However, the cross-sections of ${ }^{6} \mathrm{Li}$ and ${ }^{7} \mathrm{Be}$ are approximately same for both the reactions. The enhanced yield of ${ }^{9} \mathrm{Be}$ in the case of ${ }^{13} \mathrm{C}+{ }^{12} \mathrm{C}$ compared to ${ }^{12} \mathrm{C}+{ }^{12} \mathrm{C}$ reaction may be due to the most probable binary splitting channel, i. e. ${ }^{25} \mathrm{Mg}$ splitting into ${ }^{9} \mathrm{Be}+{ }^{16} \mathrm{O}$.

For the present study, all detected $2 \alpha$ events (for a particular angle of the telescope for both reactions) have been extracted from the inclusive event-by-event data to reconstruct the excitation energy spectra of the ${ }^{8}$ Be decay $[11,12]$. The $2 \alpha$ events originate mainly from (i) decay of particle unbound ${ }^{8} \mathrm{Be}$ states and (ii) decay of particle unbound excited states of ${ }^{12} \mathrm{C}\left({ }^{13} \mathrm{C}\right) \rightarrow 3 \alpha(+\mathrm{n})$ emission, either directly or through the sequential process ${ }^{12} \mathrm{C}\left({ }^{13} \mathrm{C}\right)$ $\rightarrow{ }^{8} \mathrm{Be}\left({ }^{9} \mathrm{Be}\right)+\alpha \rightarrow 3 \alpha(+\mathrm{n})$. The decay of ${ }^{8} \mathrm{Be}$ was identified by reconstructing the ${ }^{8} \mathrm{Be}$ excitation energy spectrum (shown in Figure 8) for all events in which two alpha particles hit two separate strips within the detector. The peaks at excitation energy $\left(\mathrm{E}_{x}\right)=0 \mathrm{MeV}$ correspond to decays of the particle-unstable ground state of ${ }^{8} \mathrm{Be}\left(\mathrm{J}^{p}=0^{+}\right)$formed in both the reactions; however the yield is more in the case of ${ }^{12} \mathrm{C}+{ }^{12} \mathrm{C}$ reaction. The pronounced bump at excitation energy $\sim 0.5 \mathrm{MeV}$ observed in the reaction ${ }^{13} \mathrm{C}+{ }^{12} \mathrm{C}$ is due to the decay of the $2.43 \mathrm{MeV}$ state in ${ }^{9} \mathrm{Be}$, which is not at all visible for ${ }^{12} \mathrm{C}+{ }^{12} \mathrm{C}$ reaction. The broad peak observed at $\mathrm{E}_{x} \sim 1.5 \mathrm{MeV}$, is due to the sequential breakup

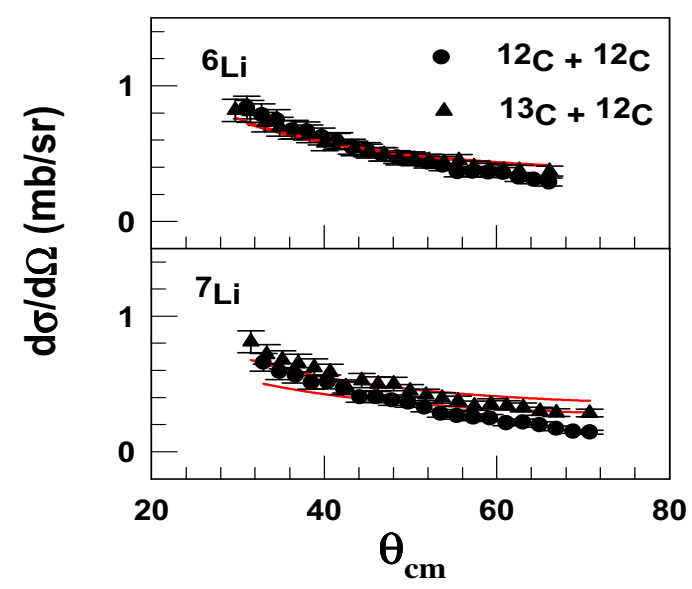

Figure 6. The $\mathrm{cm}$ angular distribution of $\mathrm{Li}$ isotopes for ${ }^{12} \mathrm{C}$ $(80 \mathrm{MeV})+{ }^{12} \mathrm{C}$ (solid circle) and ${ }^{13} \mathrm{C}(78.5 \mathrm{MeV})+{ }^{12} \mathrm{C}$ (solid triangle) reactions. The solid lines (red) are the corresponding fit with $\sim 1 / \sin \theta_{c m}$.

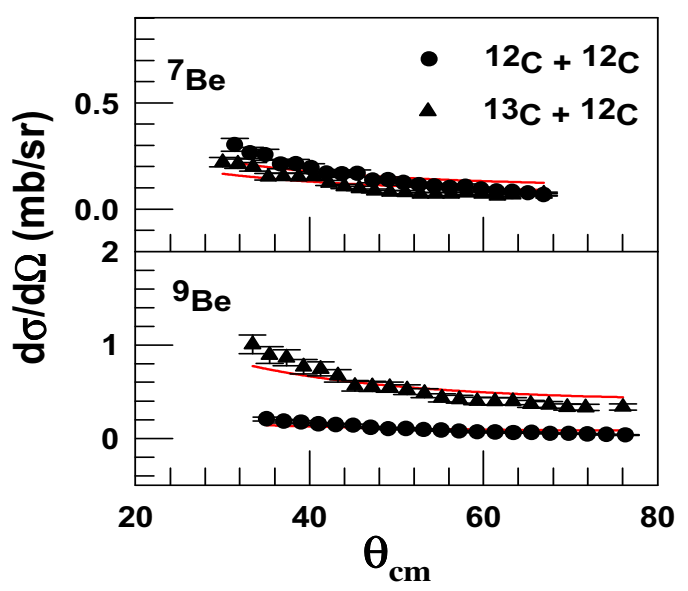

Figure 7. Same as figure 6 for isotopes of $\mathrm{Be}$ in ${ }^{12} \mathrm{C}(80 \mathrm{MeV})$ $+{ }^{12} \mathrm{C}$ (solid circle) and ${ }^{13} \mathrm{C}(78.5 \mathrm{MeV})+{ }^{12} \mathrm{C}$ (solid triangle) reactions. The solid lines (red) are the corresponding fit with $1 / \sin \theta_{c m}$.

channel of ${ }^{12} \mathrm{C}$ and is more pronounced for ${ }^{12} \mathrm{C}+{ }^{12} \mathrm{C}$ reaction. It is seen from figure 8 that there is significant difference in the cluster state formation in these two reactions and the yield of neutron rich $\mathrm{Be}$ isotope i.e. ${ }^{9} \mathrm{Be}$ is much more in ${ }^{13} \mathrm{C}+{ }^{12} \mathrm{C}$ reaction. The large cross-section ${ }^{8} \mathrm{Be} /{ }^{9} \mathrm{Be}$ in ${ }^{12} \mathrm{C}+{ }^{12} \mathrm{C} /{ }^{13} \mathrm{C}+{ }^{12} \mathrm{C}$ also support the formation of clusters as observed in references $[8,9]$. The electromagnetic transition is another probe which can be used to study the clustering effect in light nuclei [13].

\section{Conclusions}

Fragment emission mechanisms have been studied for ${ }^{12} \mathrm{C}$ $(80 \mathrm{MeV})+{ }^{12} \mathrm{C}$ and ${ }^{13} \mathrm{C}(78.5 \mathrm{MeV})+{ }^{12} \mathrm{C}$ reactions. From the preliminary analysis, it has been observed that the energy distributions of different isotopes of $\mathrm{Li}$ and $\mathrm{Be}$, obtained in the above two reactions, are found to be nearly 


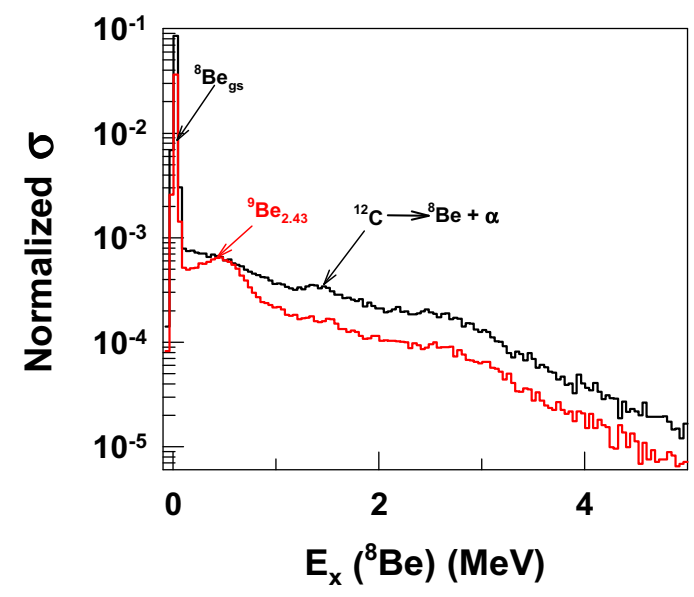

Figure 8. Excitation energy spectrum for ${ }^{8} \mathrm{Be} \rightarrow 2 \alpha$ emitted in the reactions ${ }^{12} \mathrm{C}+{ }^{12} \mathrm{C}$ (black-Solid line) and ${ }^{13} \mathrm{C}+{ }^{12} \mathrm{C}$ (redSolid line).

Gaussian in shapes with their centroid at the expected kinetic energies for the binary breakup obtained from the Viola systematic corrected by corresponding asymmetric factors. The $\mathrm{cm}$ angular distributions $(\mathrm{d} \sigma / \mathrm{d} \Omega)$, of these fragments are found to follow $\sim 1 / \sin \theta_{c m}$, which signifies the emission of these fragments are from a long lived equilibrated composite. The differential cross-sections of neutron-rich fragments, viz. ${ }^{7} \mathrm{Li}$ and ${ }^{9} \mathrm{Be}$, obtained in ${ }^{13} \mathrm{C}$ $+{ }^{12} \mathrm{C}$ reaction are found to be more than those obtained in ${ }^{12} \mathrm{C}+{ }^{12} \mathrm{C}$ reaction at each angle. On the other hand, the differential cross-sections of ${ }^{6} \mathrm{Li}$ and ${ }^{7} \mathrm{Be}$ are approximately same for both the reactions. The enhanced yield of ${ }^{9} \mathrm{Be}$ observed in the ${ }^{13} \mathrm{C}+{ }^{12} \mathrm{C}$ reactions may be due to the most probable binary splitting of the compound nucleus ${ }^{25} \mathrm{Mg}$ into ${ }^{9} \mathrm{Be}+{ }^{16} \mathrm{O}$ channel. A pronounced bump has been observed at excitation energy $\sim 0.5 \mathrm{MeV}$ in the excitation energy spectrum plot generated from $2 \alpha$ detected events for the reaction ${ }^{13} \mathrm{C}+{ }^{12} \mathrm{C}$, which is due to the decay of the $2.43 \mathrm{MeV}$ state in ${ }^{9} \mathrm{Be}$ and is not at all visible for ${ }^{12} \mathrm{C}+{ }^{12} \mathrm{C}$ reaction. It has also been seen from the $2 \alpha$ detected events that the production of neutron-rich isotope viz. ${ }^{9} \mathrm{Be}$ is more in ${ }^{13} \mathrm{C}+{ }^{12} \mathrm{C}$ compared to those produced in ${ }^{12} \mathrm{C}+{ }^{12} \mathrm{C}$ reaction. Further details analysis for these two systems are in progress.

\section{Acknowledgements}

The authors are thankful to the crew of Pelletron-linac facility for the smooth operation of the machine during the experiment.

\section{References}

[1] S. J. Sanders et al., Phys. Rep. 311, 487 (1999) and references therein.

[2] C. Beck, Y. Abe, N. Aissaoui, B. Djerroud and F. Hass, Phys. Rev. C 49, 5 (1994).

[3] D. Shapira, J. L. C. Ford, Jr., J. Gomez del Campo, R. G. Stokstad, and R. M. DeVries, Phys. Rev. Lett. 43, 1781 (1979).

[4] C. Bhattacharya et al., Phys. Rev. C 72, 021601(R) (2005).

[5] S. Kundu, A. Dey, K. Banerjee, T. K. Rana, S. Muhkopadhayay, D. Gupta, R. Saha, S. Bhattacharya, and C. Bhattacharya, Phys. Rev. C 78, 044601 (2008).

[6] S. Kundu et al., DAE Symp. On Nucl. Phys. 55, 326(2010).

[7] B. Shivakumar, S. Ayik, B. A. Harmon, and D. Shapira, Phys. Rev. C 35, 1730 (1987).

[8] Morelli L et al., J. Phys. G: Nucl. Part. Phys. 41, 075107 (2014).

[9] Morelli L et al., J. Phys. G: Nucl. Part. Phys. 41, 075108 (2014).

[10] C. Beck and A. Szanto de Toledo, Phys. Rev. C 53, 1989 (1996).

[11] T. K. Rana et al., Int. J. Mod. Phys. E 20, 789 (2011).

[12] T. K. Rana et al., Phys. Rev C 78, 027602 (2008).

[13] D. G. Jenkins, Clusters in Nuclei vol. 3 (Lecture Notes in Physics vol 875) ed C Beck (Berlin: Springer) pp 31-50 (2014). 